\title{
Original
}

\section{Effects of Zoledronic Acid on Human Gingival Fibroblasts and Human Umbilical Vein Endothelial Cells}

\author{
Yumi Kambara $^{\text {1) }}$, Eizaburo Kobayashi ${ }^{1)}$, Hiroaki Katsuragi ${ }^{2)}$ and Akira Tanaka ${ }^{1,3)}$ \\ 1) Department of Oral and Maxillofacial Surgery, The Nippon Dental University Graduate School of Life Dentistry at Niigata, Niigata, Japan \\ 2) Department of Microbiology, The Nippon Dental University Graduate School of Life Dentistry at Niigata, Niigata, Japan \\ ${ }^{3)}$ Division of Cell Regeneration and Transplantation, Advanced Research Center School of Life Dentistry at Niigata, Niigata, Japan \\ (Accepted for publication, January 27, 2021)
}

\begin{abstract}
We evaluated the effects of zoledronic acid (ZOL) on human gingival fibroblasts (HGFs) and human umbilical vein endothelial cells (HUVECs) associated with wound healing in oral soft tissues. HGFs and HUVECs were divided into two groups: a culture media control group and a group exposed to ZOL $(50 \mu \mathrm{M})$. Cell proliferation was measured after 2, 4, 6 , and 8 days. The migration ability of cells was measured for each experiment using the wound healing assay. The apoptosis rate was confirmed using the apoptosis assay. Culture supernatants were collected from each experimental group and vascular endothelial growth factor (VEGF) production in the culture media was measured using enzyme-linked immunosorbent assay (ELISA). Further, the expression level of VEGF-A was evaluated and compared using real-time quantitative polymerase chain reaction. The proliferation and migration abilities of both HGFs and HUVECs were confirmed to be suppressed by the addition of ZOL, resulting in apoptosis. ELISA revealed that the quantity of VEGF produced in HGFs was significantly higher in the ZOL group than in the control group until 2 days after the addition of ZOL. In HGFs, the mRNA expression levels of intracellular VEGF-A increased with the addition of ZOL, demonstrating the production of VEGF. In contrast, in HUVECs, although the mRNA expression levels of endogenous VEGF-A increased with the addition of ZOL, VEGF production was considerably decreased in the culture supernatant, indicating the possibility of abnormalities in the autocrine functions of endogenous VEGF or intracellular signal transduction of exogenous VEGF. These data suggest the utility of therapeutic approaches directed toward abnormalities in VEGF intracellular signaling to improve medicationrelated osteonecrosis of the jaw soft-tissue healing.
\end{abstract}

Key words: Zoledronic acid, Human gingival fibroblasts, Human umbilical vein endothelial cells, Vascular endothelial growth factor, Fibroblast growth factor, Vascular endothelial growth factor A

\section{Introduction}

Bone resorption inhibitors or bone-modifying agents are widely used to treat hypercalcemia caused by bone metastases, multiple myeloma, and malignancies as well as metabolic bone diseases, such as osteoporosis and Paget's disease.

These drugs change the balance of bone remodeling and have inhibitory effects on osteoclast activity ${ }^{1-3)}$. However, the mechanism of the pathogenesis of medication-related osteonecrosis of the jaw (MRONJ), a side effect of these drugs, has not been elucidated in detail and no preventive method or therapy has been established to date. Pathologically, MRONJ is thought to result in osteomyelitis and exacerbation due to bacterial infection and bone exposure caused by the inadequate healing of oral soft tissue after tooth extraction, in addition to ischemic osteonecrosis of $\mathrm{MRONJ}^{4-6)}$. Because bisphosphonate preparations (BPs) are representatives of bone resorption inhibitors that have a strong affinity for hydroxyapatite on the bone surface, they are absorbed by the bone surface and are taken up by osteoclasts, resulting in the apoptosis of osteoclasts ${ }^{3)}$. Additionally, studies have suggested that BPs stimulate the

Correspondence to: Dr. Yumi Kambara, Department of Oral and Maxillofacial Surgery, The Nippon Dental University Graduate School of Life Dentistry at Niigata, 1-8 Hamaura, Niigata City, Japan; TEL: +81-25-267-1500; Fax:+8125-267-9061; E-mail: yumikambara@ngt.ndu.ac.jp production of osteoprotegerin, an osteoclast differentiation inhibitor in osteoblasts, and inhibit osteoclast differentiation ${ }^{3,6}$. Moreover, BPs are known to affect fibroblasts and vascular endothelial cells ${ }^{7-11)}$; however, there are few detailed studies regarding the mechanism of BPs involved in soft-tissue healing. Therefore, the effects of zoledronic acid (ZOL) on human gingival fibroblasts (HGFs) and human umbilical vein endothelial cells (HUVECs) were evaluated in this study.

\section{Cell culture \\ Materials and Methods}

HGFs (ATCC, Manassas, USA: No. PCS-201-018) and HUVECs (Promo Cell GmbH, Heidelberg, Germany: No. C-12206) were each purchased from their respective companies. HGFs were cultured in $10 \%$ fetal bovine serum (Life Technologies Inc., Van Allen Way, CA, USA), $0.1 \%$ essential amino acid solution (Life Technologies Inc.), $1 \%$ penicillin streptomycin (Life Technologies Inc.), and Dulbecco's modified Eagle's medium (Nihon Pharmaceutical Co., Ltd., Tokyo, Japan) supplemented with $0.1 \%$ fungizone (Life Technologies Inc.). Cells were passaged 4-7 times during the experiment. HUVECs were cultured in Endothelial Cell Growth Medium 2 kit (PromoCell GmbH: No. C-22111) and subjected to 2-5 passages during the experiment. Cells were cultured in a humidified incubator set at $37^{\circ} \mathrm{C}$ and $5 \% \mathrm{CO}_{2}$. 
The experiments were conducted using a control group, in which HGFs and HUVECs were cultured alone, and a ZOL group, in which 50 $\mu \mathrm{M}$ of ZOL (Zometa ${ }^{\circledR}$, Novartis Pharma Co. Ltd., Tokyo, Japan) was added to the media.

\section{Cell proliferation assay}

For each experimental group, $1.0 \times 10^{4} \mathrm{HGFs}$ and HUVECs were seeded into $35-\mathrm{mm}$ petri dishes and cultured in their respective growth media. After seeding, the medium was changed and $50 \mu \mathrm{M}$ of $\mathrm{ZOL}$ was added at $24 \mathrm{~h}$. The cultured cells were harvested using $0.25 \%$ trypsin/ ethylenediaminetetraacetic acid (EDTA) and then stained with $0.4 \%$ trypan blue solution (Trypan Blue Stain, Gibco by Thermo Fisher Scientific Inc., Rockville, MA, USA) after 2, 4, 6, and 8 days. Viable cell counts were determined using a hemocytometer. Six treatments were administered to each experimental group.

\section{Wound healing assay}

Wound healing assays (CytoSelect ${ }^{\mathrm{TM}}$ 24-Well Wound Healing Assay, Cell Biolabs, Inc., San Diego, CA, USA) were performed to evaluate the migration abilities of HGFs and HUVECs. Inserts were placed on 24-well plates and a suspension of HGFs or HUVECs $\left(1 \times 10^{5}\right.$ cells/ well $/ 500 \mu \mathrm{l})$ was pipetted into both sides of the inserts. The cells were cultured for 24-48 h, and the inserts were removed when the cells formed a monolayer. Once the formation of a $0.9-\mathrm{mm}$ wound area was confirmed, the media was replaced with the new media and $50 \mu \mathrm{M}$ of ZOL was added. The migration ability of HGFs was measured up to 48 h every $12 \mathrm{~h}$ and that of HUVECs was measured up to $24 \mathrm{~h}$ every $8 \mathrm{~h}$. Each well was marked prior to cell seeding so that the same location could be found when photographs were taken. To visually evaluate cell migration, the distances between the wounds were measured using ImageJ (National Institutes of Health, Bethesda, MD, USA), and photographs were captured using the EVOSTM XL Cell Imaging System (Thermo Fischer Scientific Inc., MA, USA). Experiments were evaluated using the following formula:

Percent closure $(\%)=($ Length of migration field $[\mathrm{mm}] /$ Length of total wound field $[\mathrm{mm}]) \times 100$

Each sample was randomly measured in five different regions, and measurements were performed three times in each experimental group.

\section{Apoptosis assay}

Apoptosis assays were performed to assess the apoptosis rates of HGFs and HUVECs. For each experimental group, $1.0 \times 10^{4} \mathrm{HGFs}$ and HUVECs were seeded into $35-\mathrm{mm}$ petri dishes and cultured in their respective growth media. After seeding, the medium was changed and 50 $\mu \mathrm{M}$ of ZOL was added at $24 \mathrm{~h}$. The cells were harvested via EDTA treatment at 1,2, and 4 days, and cell suspensions were stained with Muse $^{\circledR}$ Annexin V \& Dead Cell Reagent (No. MCH100105, Merck, Darmstadt, Germany) for 20 min. The Muse ${ }^{\circledR}$ Cell Analyzer (Merck) was used to assess apoptosis.

\section{Enzyme-linked immunosorbent assay (ELISA)}

To investigate the effects of ZOL addition on the growth factors produced by HGFs and HUVECs, a comparative study was conducted using ELISA, in which $1.0 \times 10^{4}$ cells of each type were seeded into 35$\mathrm{mm}$ petri dishes and cultured for $24 \mathrm{~h}$. After cell adhesion was confirmed, the media was replaced with each control medium alone or with a medium supplemented with $50 \mu \mathrm{M}$ of ZOL. In HGFs, the supernatant was collected at 1,2, and 3 days after exposure, followed by the measurement of the expression levels of vascular endothelial growth factor (VEGF). In HUVECs, the VEGF concentration was measured every $6 \mathrm{~h}$ for $24 \mathrm{~h}$ after exposure. Measurements were conducted using the Human VEGF ELISA Kit (R\&D Systems, Minneapolis, MN, USA) and were performed six times for each experimental group.

\section{Real-time quantitative polymerase chain reaction (qPCR) analysis}

Among the VEGF families, VEGF-A is most involved in angiogenesis. Following evaluation by ELISA, the expression level of VEGF-A in individual cells was evaluated over time using a qPCR method. To determine the primers for this study, the mRNA expression of VEGF-A was first confirmed in individual cells. Each cell type was seeded into a 60-mm petri dish and cultured in their respective culture medium. Total RNA was extracted from each cell type according to the ISOGENII (Nippon Gene, Co., Ltd., Tokyo, Japan) protocol, and $1 \mu \mathrm{g}$ of the total RNA extracted was used for the synthesis of cDNA using the High Capacity cDNA Reverse Transcription kit (Life Technologies, Carlsbad, CA, USA). VEGF-A and Platinum PCR Super Mix (Life Technologies) were used and PCR amplification was performed using the following PCR conditions: 35 cycles of denaturation at $94^{\circ} \mathrm{C}$ for $30 \mathrm{~s}$, annealing at $55^{\circ} \mathrm{C}$ for $30 \mathrm{~s}$, and extension at $72^{\circ} \mathrm{C}$ for $60 \mathrm{~s}$. PCR amplification using glyceraldehyde-3-phosphate dehydrogenase (GAPDH) as an endogenous control was performed under the same conditions (Table 1). The amplified PCR products were electrophoresed on a $2 \%$ agarose gel (Nippon Gene) supplemented with ethidium bromide and observed under ultraviolet (UV) irradiation to confirm the bands. Because the mRNA expression of VEGF-A was confirmed in each cell using the qPCR method, PCR expression levels after the addition of ZOL were compared using the qPCR method. In HGFs, the mRNA expression level of VEGF-A was measured 1, 2, and 3 days after the media was changed and ZOL was added. In HUVECs, the mRNA expression level of VEGF-A was measured at $6,12,18$, and $24 \mathrm{~h}$. Total RNA was extracted in the same manner as described above, and cDNA was synthesized using the PrimeScript ${ }^{\mathrm{TM}}$ RT Reagent kit with gDNA Eraser (Perfect Real Time) (Takara Clontech, Kusatsu, Japan). Thereafter, reaction solutions were prepared using the Fast $\mathrm{SYBR}^{\circledR}$ Green Master Mix (Applied Biosystems, Foster City, CA, USA), and qPCR was performed using Step One Plus ${ }^{\mathrm{TM}}$ (Applied Biosystems). DNA polymerase was activated at $95^{\circ} \mathrm{C}$ for $20 \mathrm{~s}$, denaturation was set at $95^{\circ} \mathrm{C}$ for $3 \mathrm{~s}$, and annealing/extension was set at $60^{\circ} \mathrm{C}$ for $30 \mathrm{~s}$; each step was repeated for

Table 1. Primer sequences for qRT-PCR

\begin{tabular}{cllcc}
\hline Gene & & \multicolumn{1}{c}{ Primer Sequence } & Product Size (bp) & GenBank accession number \\
\hline VEGF-A & Forward & 5'-CCCTGATGAGATCGAGTACA-3' & 174 & NM_001025366.3 \\
& Reverse & 5'-AGGAAGCTCATCTCTCCTAT-3' & & \\
\hline GAPDH & Forward & 5-CCCACTCCTCCACCTTTGAC-3 & 100 & NM_001357943.2 \\
& Reverse & 5-CTGTTGCTGTAGCCAAATTCGT-3' & & \\
\hline
\end{tabular}


40 cycles. This was performed six times for each experimental cell type, and the comparative $\mathrm{CT}$ method $(\Delta \Delta \mathrm{CT})$ was used for analysis.

\section{Statistical analysis}

Data were analyzed using BellCurve ${ }^{\circledR}$ for Excel (Social Survey Research Information Co., Ltd., Tokyo, Japan), and the Mann-Whitney U test or Student's $t$-test were used to compare the two groups. Data are expressed as mean \pm standard error, and $\mathrm{p}<0.05$ was considered statistically significant.

\section{Results}

\section{Cell proliferation assay}

Each cell type in the ZOL group was compared with that in the culture media control group. The results showed that HGFs and HUVECs in the ZOL group had a significantly lower cell proliferation rate than those in the control group ( $\mathrm{p}<0.01)$. The number of viable cells increased until 2 days after the addition of ZOL but decreased thereafter (Fig. 1a, b). In particular, compared with the control group, HGFs showed a $45.0 \%$ decrease in proliferation after 2 days and a $98.6 \%$ decrease after 8 days in the ZOL group. In HUVEC, the growth number decreased by $88.0 \%$ after 2 days and by $99.1 \%$ after 8 days in the ZOL group.

\section{Wound healing assay}

The migration abilities of HGFs and HUVECs were compared between the control and ZOL groups. A significant decrease in the migration ability of HGFs was observed in the ZOL group compared with that of HGFs in the control group at each measurement time point every 12 a

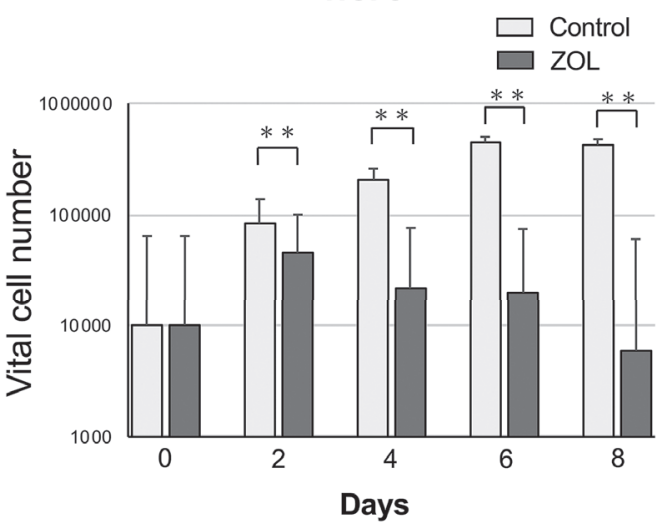

b

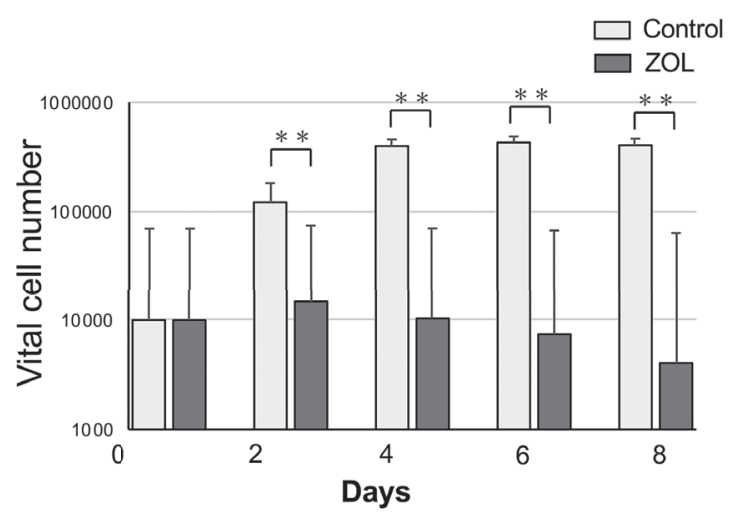

Figure 1. Cell proliferation assay: There were significant differences in the numbers of proliferating (a) HGFs and (b) HUVECs in the control and ZOL groups in the cell proliferation assay $(* * p<0.01)$.
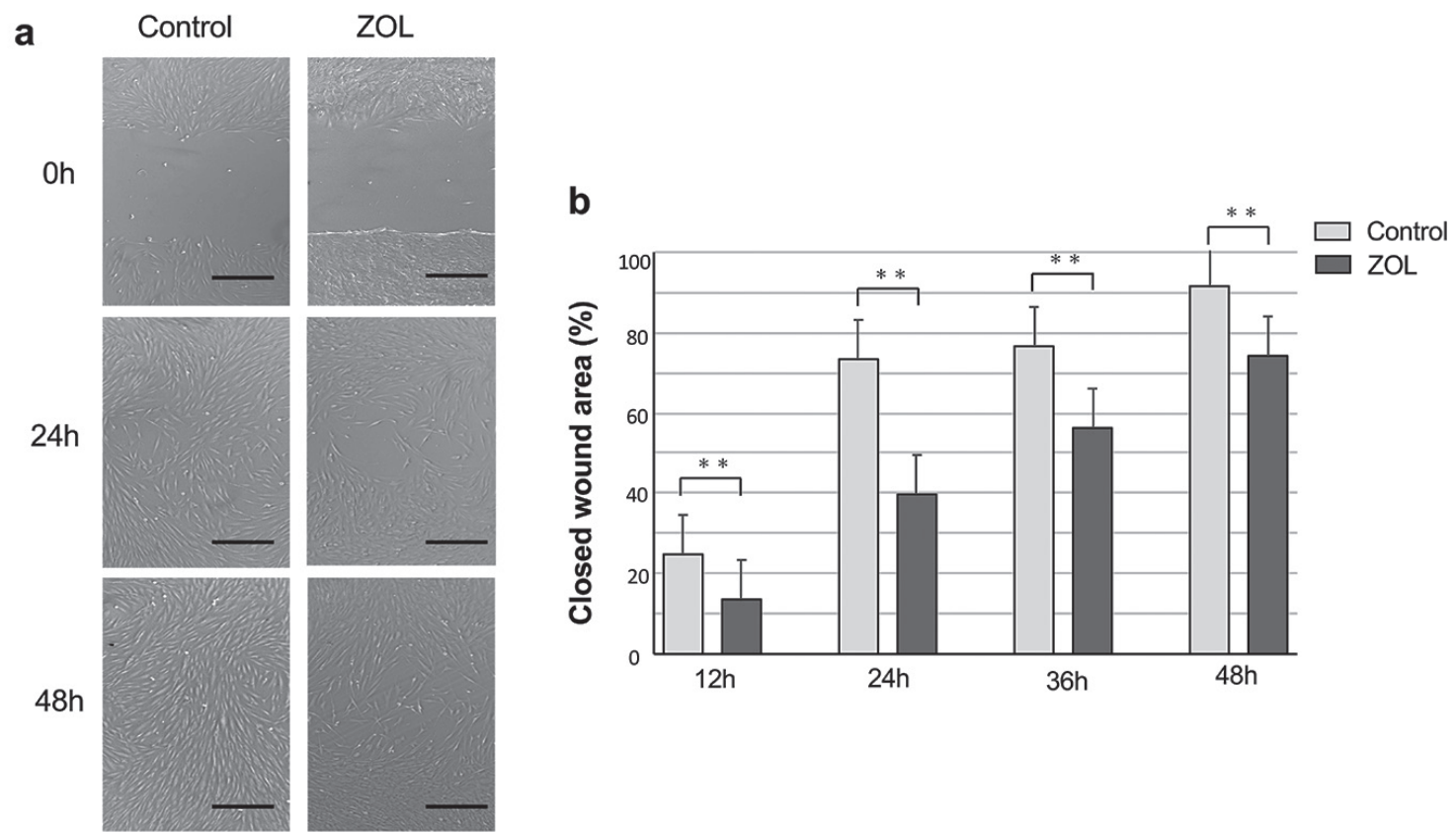

Figure 2. Wound healing assay in HGFs: (a) Microscopic images (b) showed a significant decrease in the migration ability of cells in the ZOL group compared with that of cells in the control group when measured every $12 \mathrm{~h}(* * \mathrm{p}<0.01)$. (Scale bar $=500 \mu \mathrm{m})$. 
$\mathrm{h}(\mathrm{p}<0.01)$ (Fig. 2a, b). In contrast a decrease in the migration ability of HUVECs was observed at each measurement time point every $8 \mathrm{~h}(\mathrm{p}$ $<0.01$ ) (Fig. 3a, b). The migration rate of HGFs decreased by $33 \%$ after $24 \mathrm{~h}$ and by $17.3 \%$ after $48 \mathrm{~h}$ in the ZOL group compared with that in the control group. On the other hand, after 24 hours, the migration rate if HUVECs decreased by $27.5 \%$ in the ZOL group compared with that in the control group.

\section{Apoptosis assay}

The apoptosis rates of HGFs and HUVECs in the control and ZOL groups were compared. Both HGFs and HUVECs in the ZOL group showed significant apoptosis on day $4(\mathrm{p}<0.01)$ (Fig. $4 \mathrm{a}-\mathrm{d})$. On day 4 , HGFs showed a $6.4 \%$ increase in apoptosis in the ZOL group compared with that in the control group. Similarly, on day 4, HUVECs showed a $6.8 \%$ increase in apoptosis the ZOL group compared with that in the

a Control

ZOL

Oh

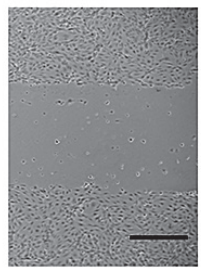

$8 \mathrm{~h}$

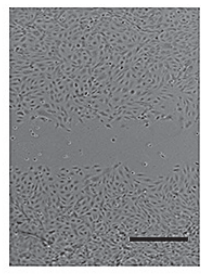

$24 h$



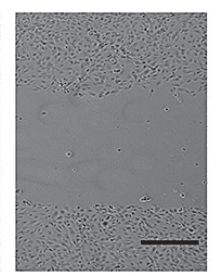
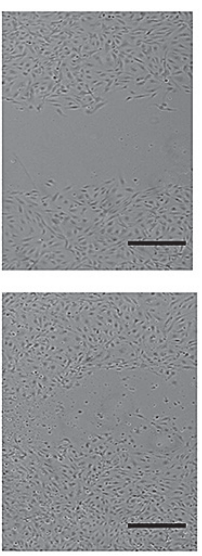



Figure 3. Wound healing assay in HUVECs: (a) Microscopic images (b) showed reductions in the migration ability of cells in the ZOL group compared with that of cells in the control group at all measurement time points taken every 8 hours $(* * \mathrm{p}<0.01)$. (Scale bar $=500 \mu \mathrm{m})$.

a

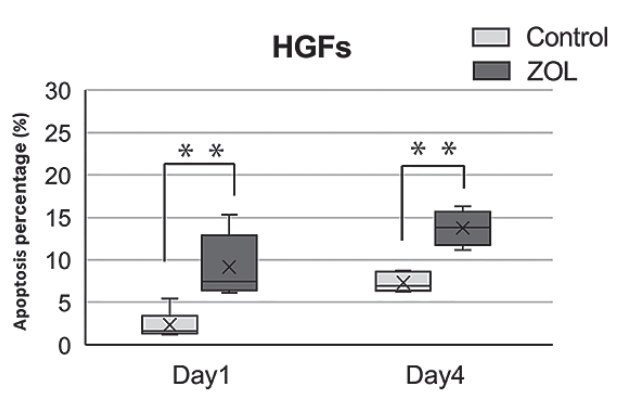

b

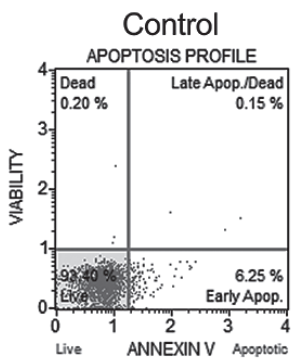

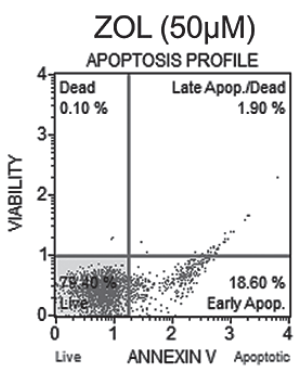

C



d

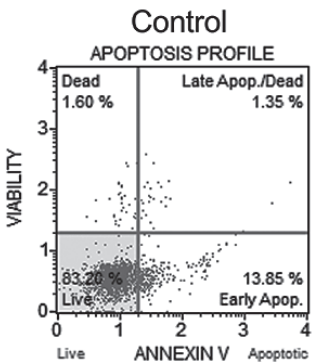

Figure 4. Apoptosis assay in HGFs and HUVECs: (a) Apoptosis in HGFs. Apoptosis was induced at 1, 2, and 4 days after ZOL exposure $(* * p<$ $0.01,{ }^{*} \mathrm{p}<0.05$ ). (b) Flow cytometric analyses of apoptotic HGFs using annexin V. (c) Apoptosis in HUVECs. Significant apoptosis was observed at 4 days after ZOL exposure $(* * p<0.01, * p<0.05)$. (d) Flow cytometric analyses of apoptotic HUVECs using annexin V. 
a

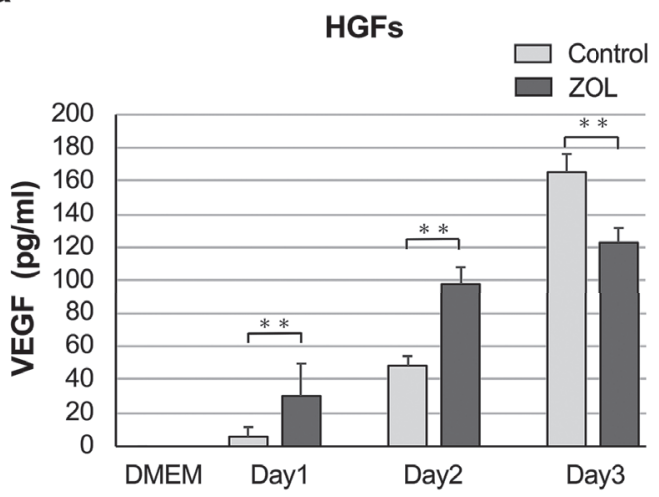

b

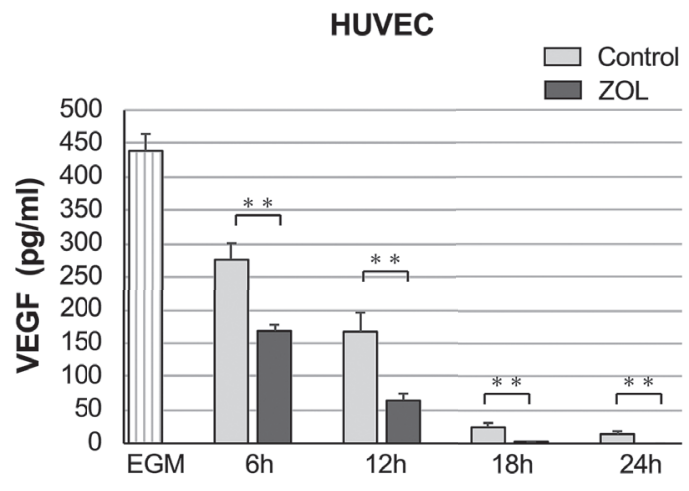

Figure 5. VEGF secretion in HGFs and HUVECs measured via ELISA: (a) In HGFs, VEGF levels increased over time in both the control and ZOL groups. (b) In HUVECs, VEGF levels significantly decreased in the culture supernatant of cells in the ZOL group compared with those in the culture supernatant of cells in the control group $(* * \mathrm{p}<0.01)$.

a

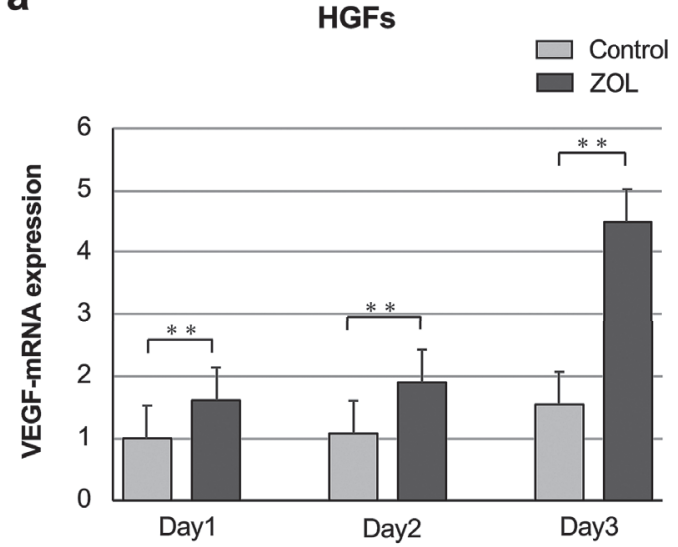

b



Figure 6. Comparison of the mRNA expression levels of VEGF in HGFs and HUVECs via real-time quantitative polymerase chain reaction: (a) In HGFs, the mRNA expression level of VEGF significantly increased in the ZOL group compared with that in the control group $(* * p<0.01)$. (b) In HUVECs, the mRNA expression level of VEGF increased $6 \mathrm{~h}$ after ZOL exposure $\left({ }^{*} \mathrm{p}<0.05\right)$.

control group.

\section{ELISA}

VEGF production in HGFs and HUVECs were compared using ELISA. In the HGF culture supernatant, VEGF production increased over time in both experimental groups. VEGF production in the ZOL group was significantly higher than that in the control group until 2 days after ZOL exposure but decreased on day $3(\mathrm{p}<0.01)$ (Fig. 5a). In contrast, VEGF production in the HUVEC culture supernatant tended to decrease over time in each experimental group. Furthermore, VEGF production in culture supernatants significantly decreased in the ZOL group compared with that in the control group $(\mathrm{p}<0.01)$ (Fig. 5b).

\section{qPCR analysis}

Because significant changes were observed in HGFs and HUVECs via ELISA, the expression levels of VEGF-A were compared and evaluated using qPCR. In HGFs, the mRNA expression level of VEGF-A increased up to 3 days after the addition of ZOL ( $p<0.01)$ (Fig. 6a). In contrast, in HUVECs, the mRNA expression levels of VEGF-A significantly increased up to $6 \mathrm{~h}$ after the addition of ZOL (p<0.05) (Fig. 6b).

\section{Discussion}

ZOL has a potent inhibitory effect on osteoclasts and a high affinity for bone minerals, leading to the longest duration of activity among $\mathrm{BPs}^{12)}$. Studies have suggested that ZOL induces the inhibition of the proliferation and migration of epithelial cells, HGFs, and HUVECs, possibly adversely affecting wound healing ${ }^{10,13,14)}$. However, the effects of ZOL on the production and intracellular molecular mechanisms of cytokines involved in wound healing, such as VEGF and FGF-2, have not been well documented in oral soft-tissue cells.

ZOL inhibits farnesyl pyrophosphate synthase in the mevalonate pathway of osteoclasts, thereby interfering with geranylgeraniol $(\mathrm{GGOH})$ biosynthesis and leading to osteoclast inactivity and apopto$\operatorname{sis}^{12,15)}$. The same pathway is inhibited in HGFs and HUVEC as osteoclasts, thereby leading to decreased survival and migration ability; these conditions improve with the addition of $\mathrm{GGOH}^{16,17)}$. In this study, HGFs and HUVECs also showed significant apoptosis 4 days after the addition of ZOL, suggesting that the mevalonate pathway was inhibited.

In vitro studies have shown that the concentration of ZOL that most effectively inhibits osteoclasts is $100 \mu \mathrm{M}$, with no effects observed at concentrations less than $1 \mu \mathrm{M}$; however, the response does not increase 
in a concentration-dependent manner ${ }^{18)}$. Moreover, the ZOL concentration used in clinical practice is pharmacologically equivalent to approximately $1-5 \mu \mathrm{M}$, and ZOL concentration in bone tissue reportedly increases 100 -fold owing to the preferential transfer of ZOL to the bone ${ }^{18,19)}$. There are limited data regarding the effects of clinically relevant ZOL concentrations on soft-tissue cells, and the extent to which it affects HGFs and HUVECs remains unknown. Because HGFs and HUVECs are significantly apoptotic when the ZOL concentration is $>50 \mu \mathrm{M}^{10,20,21)}$, experiments were performed at $50 \mu \mathrm{M}$ in the present study.

Angiogenesis plays an important role in the proliferative phase of wound healing. Although the formation of granulation tissue with active angiogenesis is crucial for curing tooth extraction sockets, it is widely known that cytokines and chemokines are intricately involved in the mechanism of action ${ }^{22)}$. VEGF is one of the most potent angiogenesis-promoting factors involved in angiogenesis, inflammatory response, and granulation tissue formation ${ }^{23)}$.

VEGF production is low in human skin fibroblasts under normal conditions, and stressors, such as hypoxia, TGF- $\beta$, UV radiation, prostaglandin E2, and IL-4, have been reported to enhance VEGF produc$\operatorname{tion}^{24-26)}$. In osteoblasts, statins that inhibit the mevalonate pathway, similar to ZOL, stimulate a negative feedback mechanism that increases the mRNA and protein expression levels of VEGF-A by inhibiting Ras and Rho protein syntheses, which are crucial for cellular differentiation and proliferation occurring downstream of the pathway, leading to angiogenesis $^{27}$. Similarly, the addition of ZOL also leads to the production of VEGF-A mRNA in fibroblasts, suggesting a similar pathway ${ }^{28,29)}$. In this study, ZOL exposure also increased VEGF production and VEGF-A mRNA levels in HGFs at 24 and $48 \mathrm{~h}$, demonstrating consistent results with those of previous studies. However, after $72 \mathrm{~h}$, the mRNA expression level of VEGF-A was increased compared with those in the control group. In contrast, the VEGF levels decreased in the culture supernatant, as determined using ELIZA. We considered that the amount of VEGF produced in the culture supernatant turned to decrease, reflecting the decrease in the number of viable cells and increase in apoptosis.

Although HUVECs have autocrine effects via autocrine endogenous VEGF, VEGF in these cells do not affect angiogenic responses and only contribute to autosurvival. The autocrine signals of such VEGFs have been reported to be strongly induced by cellular stressors, such as hypoxia, radiation, and reactive oxygen species ${ }^{30,31)}$. In this study, the mRNA expression level of VEGF-A significantly increased at $6 \mathrm{~h}$. This increase is possibly due to cell stress caused by the addition of ZOL. However, ELISA revealed that the amount of VEGF in the culture supernatant of cells in the ZOL group significantly decreased at 6 and $12 \mathrm{~h}$ and that no amount of VEGF was observed after $18 \mathrm{~h}$. The tendency of the amount of VEGF to decrease in the culture supernatant from $6 \mathrm{~h}$ and the increase in the mRNA expression level of VEGF-A are roughly conflicting. It has been suggested that the mRNA expression level of VEGF-A transiently increases due to cell stress caused by ZOL. On the other hand, the reason for the decrease in the amount of VEGF in the culture supernatant was considered to be due to abnormalities in the autocrine function of endogenous VEGF or intracellular signal transduction of exogenous VEGF taken up intracellularly.

Although the paracrine secretion of exogenous VEGF is involved in angiogenesis and cell proliferation ${ }^{27)}$, protein kinase B(AKT), extracellular signal-regulated kinase, and caspase- 3 signaling pathways are inhibited by ZOL exposure and are involved in the promotion of apoptosis and inhibition of cell proliferation ${ }^{21}$. In addition, this study suggests that VEGF incorporated into HUVECs could not function due to the inhibition of these signal transductions via the addition of ZOL, possibly in- hibiting angiogenesis and cell proliferation.

Based on the results obtained in this study, ZOL was demonstrated to suppress the cell proliferation and migration abilities of HGFs and HUVECs, leading to apoptosis. In addition, although ZOL promotes VEGF production in HGFs, ZOL may inhibit the autocrine function of endogenous VEGF and the intracellular signal transduction of exogenous VEGF in HUVECs.

The number of patients with MRONJ is expected to increase in the future and there is an urgent need to establish pharmacological and preventive treatments in addition to surgical treatments currently considered effective. The effects of various cytokines on intracellular signal transduction pathways in response to ZOL and the development of therapies that normalize cytokines causing functional abnormalities in response to ZOL require further investigation.

In conclusion, the proliferation and migration ability of both HGFs and HUVECs were confirmed to be suppressed by the addition of ZOL, resulting in apoptosis. The mRNA expression levels of intracellular VEGF-A were confirmed to increase with the addition of ZOL in HGFs, and HGFs were shown to produce VEGF. Conversely, in HUVECs, although the mRNA expression level of endogenous VEGF-A increased with the addition of ZOL, VEGF levels were considerably decreased in the culture supernatant, The results indicate the possibility of abnormalities in the autocrine function of endogenous VEGF or intracellular signal transduction of exogenous VEGF taken up intracellularly.

\section{Acknowledgments}

We would like to extend our gratitude to Dr. Yukio Miyagawa who is a specially appointed professor at The Nippon Dental University for providing guidance for this study.

\section{Conflict of Interest}

The authors declare that they have no conflicts of interest.

\section{References}

1. Knight RJ, Reddy C, Rtshiladze MA, Lvoff G, Sherring D and Marucci D. Bisphosphonate-related osteonecrosis of the jaw: tip of the iceberg. J Craniofac Surg 21: 25-32, 2010

2. Migliorati CA, Siegel MA and Elting LS. Bisphosphonate-associated osteonecrosis: a long-term complication of bisphosphonate treatment. Lancet Oncol 7: 508-514, 2006

3. Michaelson MD and Smith MR. Bisphosphonates for treatment and prevention of bone metastases. J Clin Oncol 23: 8219-8224, 2005

4. Ristow O, Gerngroß C, Schwaiger M, Hohlweg-Majert B, Kehl V, Jansen H, Hahnefeld L, Otto S and Pautke C. Is bone turnover of jawbone and its possible over suppression by bisphosphonates of etiologic importance in pathogenesis of bisphosphonate-related osteonecrosis? J Oral Maxillofac Surg 72: 903-910, 2014

5. Uyanne J, Calhoun CC and Le AD. Antiresorptive drug-related osteonecrosis of the jaw. Dent Clin North Am 58: 369-384, 2014

6. Marx RE. Pamidronate (Aredia) and zoledronate (Zometa) induced avascular necrosis of the jaws: a growing epidemic. J Oral Maxillofac Surg 61: 1115-1117, 2003

7. Cozin M, Pinker BM, Solemani K, Zuniga JM, Dadaian SC, Cremers S, Landesberg R and Raghavan S. Novel therapy to reverse the cellular effects of bisphosphonates on primary human oral fibroblasts. J Oral Maxillofac Surg 69: 2564-2578, 2011

8. Misso G, Porru M, Stoppacciaro A, Castellano M, De Cicco F, Leonetti C, Santini D and Caraglia M. Evaluation of the in vitro and in vivo antiangiogenic effects of denosumab and zoledronic acid. Can- 
cer Biol Ther 13: 1491-1500, 2012

9. De Colli M, Zara S, di Giacomo V, Patruno A, Marconi GD, Gallorini $\mathrm{M}$, Zizzari VL, Tetè $\mathrm{G}$ and Cataldi $\mathrm{A}$. Nitric oxide-mediated cytotoxic effect induced by zoledronic acid treatment on human gingival fibroblasts. Clin Oral Investig 19: 1269-1277, 2015

10. Jung J, Park JS, Righesso L, Pabst AM, Al-Nawas B, Kwon YD and Walter C. Effects of an oral bisphosphonate and three intravenous bisphosphonates on several cell types in vitro. Clin Oral Investig 22: 2527-2534, 2018

11. Ziebart T, Halling F, Heymann P, Neff A, Blatt S, Jung J, Pabst A, Righesso L and Walter C. Impact of soft tissue pathophysiology in the development and maintenance of bisphosphonate-related osteonecrosis of the jaw (BRONJ). Dent J (Basel) 4: 36, 2016

12. Russell RG, Xia Z, Dunford JE, Oppermann U, Kwaasi A, Hulley PA, Kavanagh KL, Triffitt JT, Lundy MW, Phipps RJ, Barnett BL, Coxon FP, Rogers MJ, Watts NB and Ebetino FH. Bisphosphonates: an update on mechanisms of action and how these relate to clinical efficacy. Ann N Y Acad Sci 1117: 209-257, 2007

13. Basso FG, Pansani TN, de Oliveira CF, Turrioni AP, Soares DG, Hebling $\mathrm{J}$ and Costa CA. Cytotoxic effects of zoledronic acid on human epithelial cells and gingival fibroblasts. Braz Dent J 24: 551558, 2013

14. Wang Q, Liu J, Guo T, Liu D and Pan J. Epidermal growth factor reverses the inhibitory effects of the bisphosphonate, zoledronic acid, on human oral keratinocytes and human vascular endothelial cells in vitro via the epidermal growth factor receptor (EGFR)/Akt/ phosphoinositide 3-kinase (PI3K) signaling pathway. Med Sci Monit 25: 700-710, 2019

15. Basi DL, Lee SW, Helfman S, Mariash A and Lunos SA. Accumulation of VEGFR2 in zoledronic acid-treated endothelial cells. Mol Med Rep 3: 399-403, 2010

16. Hagelauer N, Ziebart T, Pabst AM and Walter C. Bisphosphonates inhibit cell functions of HUVECs, fibroblasts and osteogenic cells via inhibition of protein geranylgeranylation. Clin Oral Investig 19: 1079-1091, 2015

17. Ziebart T, Koch F, Klein MO, Guth J, Adler J, Pabst A, Al-Nawas B and Walter C. Geranylgeraniol - a new potential therapeutic approach to bisphosphonate associated osteonecrosis of the jaw. Oral Oncol 47: 195-201, 2011

18. Li P, Zhao Z, Wang L, Jin X, Shen Y, Nan C and Liu H. Minimally effective concentration of zoledronic acid to suppress osteoclasts in vitro. Exp Ther Med 15: 5330-5336, 2018

19. Chen T, Berenson J, Vescio R, Swift R, Gilchick A, Goodin S, LoRusso P, Ma P, Ravera C, Deckert F, Schran H, Seaman J and Skerjanec A. Pharmacokinetics and pharmacodynamics of zoledronic acid in cancer patients with bone metastases. J Clin Pharmacol
42: 1228-1236, 2002

20. Walter C, Klein MO, Pabst A, Al-Nawas B, Duschner H and Ziebart T. Influence of bisphosphonates on endothelial cells, fibroblasts, and osteogenic cells. Clin Oral Investig 14: 35-41, 2010

21. Lang M, Zhou Z, Shi L, Niu J, Xu S, Lin W, Chen Z and Wang Y. Influence of zoledronic acid on proliferation, migration, and apoptosis of vascular endothelial cells. Br J Oral Maxillofac Surg 54: 889893, 2016

22. Reinke JM and Sorg H. Wound repair and regeneration. Eur Surg Res 49: 35-43, 2012

23. Eming SA, Brachvogel B, Odorisio T and Koch M. Regulation of angiogenesis: wound healing as a model. Prog Histochem Cytochem 42: 115-170, 2007

24. Trompezinski S, Denis A, Vinche A, Schmitt D and Viac J. IL-4 and interferon-gamma differentially modulate vascular endothelial growth factor release from normal human keratinocytes and fibroblasts. Exp Dermatol 11: 224-231, 2002

25. Trompezinski S, Pernet I, Schmitt D and Viac J. UV radiation and prostaglandin E2 up-regulate vascular endothelial growth factor (VEGF) in cultured human fibroblasts. Inflamm Res 50: 422-427, 2001

26. Trompezinski S, Pernet I, Mayoux C, Schmitt D and Viac J. Transforming growth factor-beta1 and ultraviolet A1 radiation increase production of vascular endothelial growth factor but not endothelin-1 in human dermal fibroblasts. Br J Dermatol 143: 539-545, 2000

27. Maeda T, Kawane T and Horiuchi N. Statins augment vascular endothelial growth factor expression in osteoblastic cells via inhibition of protein prenylation. Endocrinology 144: 681-692, 2003

28. Zafar S, Coates DE, Cullinan MP, Drummond BK, Milne T and Seymour GJ. Zoledronic acid and geranylgeraniol regulate cellular behaviour and angiogenic gene expression in human gingival fibroblasts. J Oral Pathol Med 43: 711-721, 2014

29. Ohlrich EJ, Coates DE, Cullinan MP, Milne TJ, Zafar S, Zhao Y, Duncan WD and Seymour GJ. The bisphosphonate zoledronic acid regulates key angiogenesis-related genes in primary human gingival fibroblasts. Arch Oral Biol 63: 7-14, 2016

30. Namiki A, Brogi E, Kearney M, Kim EA, Wu T, Couffinhal T, Varticovski L and Isner JM. Hypoxia induces vascular endothelial growth factor in cultured human endothelial cells. J Biol Chem 270: 31189-31195, 1995

31. Lee S, Chen TT, Barber CL, Jordan MC, Murdock J, Desai S, Ferrara N, Nagy A, Roos KP and Iruela-Arispe ML. Autocrine VEGF signaling is required for vascular homeostasis. Cell 130: 691-703, 2007 
J.Hard Tissue Biology Vol. 30(2): 123-130, 2021 